\title{
High-capacity wave energy conversion by multi-float, multi-PTO, control and prediction: generalised state-space modelling with linear optimal control and arbitrary headings
}

\author{
Zhijing Liao, Peter Stansby, Guang Li, Senior Member, IEEE, Efrain Carpintero Moreno
}

\begin{abstract}
Wave energy converters with capacity similar to, or greater than, wind turbines are desirable for the supply of electricity to the grid. It is shown that this may be provided by multiple floats in a hinged raft-type configuration with multimode forcing. The case analysed has 8 floats and 4 power take off (PTO) units. Analysis is based on linear diffraction-radiation modelling, validated in wave basin experiments with a smaller number of floats. Control is desirable to improve energy capture, mainly demonstrated for point absorbers, but this has not previously been applied to such a complex problem with many degrees of freedom. The linear hydrodynamic model in a state-space form makes it possible to implement advanced control algorithms in real time. Linear non-causal optimal control (LNOC) is applied with wave force prediction from auto-regression. For the design case with zero heading, as the configuration heads naturally into the wave direction, energy capture is improved by between $21 \%$ and $83 \%$. The energy capture is about $62 \%$ the maximum possible from idealised analyses. Off-design, non-zero headings are also analysed to indicate how energy capture can be reduced; the contribution from different modes of forcing varies with heading and energy capture is always improved by control, by several times at $90^{\circ}$ heading.
\end{abstract}

\section{INTRODUCTION}

Ocean wave power has a worldwide average of about 2 TW [1] which is similar to wind and largely unexploited. Wave energy converter (WEC) device capacity has been much smaller than wind turbines [2], and with higher levelised cost of electricity (LCOE). This paper addresses the gap in knowledge about how to enable WECs to have capacity similar to, desirably greater than, wind turbines while optimising energy capture by control of power-take-off (PTO). This high capacity may be achieved by advancing and combining two key technologies: 1) integration of multiple floats and multiple PTO systems into one converter to improve the energy output by many times compared to conventional single PTO converters; 2) design of advanced linear optimal control which is possible if the converter exhibits linear hydrodynamics. Optimal controller design for such a complex converter with many freedoms is much more challenging than the existing

Zhijing Liao (z.liao@qmul.ac.uk) and Guang Li (g.li@qmul.ac.uk) are with the School of Engineering and Material Science, Queen Mary University of London, London, E1 4NS, UK.

Peter Stansby (p.k.stansby@manchester.ac.uk) is with the School of Engineering, University of Manchester, Manchester, M13 9PL, UK.

Corresponding author: Guang Li (g.li@qmul.ac.uk) optimal control designs for conventional converters with only one PTO system.

The desired linear hydrodynamic characteristics are embodied in the M4 (moored, multi-float, multi-mode) system which is of raft-type in modular form, initially with a bow and mid float connected by a beam, with a hinge above the mid float connected by a beam to the stern float [3], [4]. The floats are of circular cross section with vertical axes (in still water). The bow float is moored, typically to a single point mooring, and the floats increase in diameter and draft from bow to mid to stern so that wave drift forces facilitate the system to head naturally into waves. Different drafts provide natural frequencies which cover the wave frequencies of an intended site providing broad band power capture. The beam lengths are similar to half a wavelength, so that floats respond predominantly in anti-phase and again may be of different lengths to cover a range of wave frequencies. The floats have rounded or hemi-spherical bases to minimise drag losses and the freeboard is sufficiently high to avoid losses due to sloshing over the deck. This configuration has been simulated extensively using linear diffraction/radiation timedomain modelling based on Cummins' method with validation in wave basin tests, mainly in irregular waves [5].

All testing was done with a linear damper and power take off (PTO) and power capture has been improved by modelling with control [6], [7]. While there has been considerable research on control for point absorbers, increasing power capture by up to a factor of 4 [8], complex advanced control with several modes of forcing with multiple freedoms is more challenging but possible with a validated linear model [5]. It should be mentioned that the linear hydrodynamic modelling is also valid in extreme waves with large response, investigated without a damper [9], [10], further justifying use with control which generally increases response. In our initial work the linear non-causal optimal control (LNOC) was applied, with the hydrodynamic model converted to state-space form, assuming a known wave field, in effect assuming perfect wave force (more convenient than wave field) prediction [6]. This improved power capture by between $40 \%$ and $100 \%$, from lower to higher peak wave periods. Extending this to include wave force prediction using auto-regression (AR) gave slightly less improvement with predictions accurate to around two peak periods forward of a given time [7].

In order to increase power capture the float number was 
increased to 3 mid floats and 2 stern floats with two PTOs, termed a 1-3-2 configuration, while the former was 1-1-1 [5], [10]. This was modelled and tested in a wave basin again with linear dampers [10], [11] and in this case the model surprisingly slightly underestimated power capture; this is surprising since nonlinear effects normally reduce response and power capture. The reason is presently unknown although small reflections in a wave basin could be influential with reflection from radiated waves as well as directly from the beach; as the power capture increases the radiated wave power also increases. The maximum capture widths were over one wavelength for that associated with frequency at the centroid of the wave spectrum. Importantly the model does not overestimate power capture compared to wave basin tests. The modelling also showed that angular response and power capture were virtually unchanged by inclusion of a mooring.

It is clearly desirable to increase power capture further and this may be achieved with more floats, up to 8 were modelled in a 1-3-4 configuration (1 bow float, 3 mid floats, 4 stern floats and 4 PTOs) in [5]. The annual energy capture was calculated from scatter diagrams for various sites, showing annual average power of $1.25 \mathrm{MW}$ for a west UK site (Wavehub), $3 \mathrm{MW}$ for west Ireland (Belmullet) and $6 \mathrm{MW}$ for northern Spain (Death coast), corresponding to capacities of $3.75,9$ and $18 \mathrm{MW}$ respectively, now similar to planned offshore wind turbine capacities. The device size was optimised to suit the wave climate and a linear damper was applied. The largest overall length is now similar to the tip blade height of an offshore wind turbine above sea level. Such promising power capture can be improved further by the control methods (LNOC+AR) developed for the 1-1-1 system. However there are now many more freedoms and the control must work in real time to be of practical value. The multiple PTOs are coordinated by a central optimal controller to maximize the sum of energy outputs of all PTOs, complicating controller design beyond that for a single PTO [6], [7], increasing power generation and reducing LCOE.

In addition it has been assumed so far that the system aligns with the wave direction which has been confirmed in wave basin tests without a current. The system may veer away from this due to currents and windage, although currents are small in ocean conditions and wind is often aligned with the wave direction. Nevertheless the effect should be quantified, and is undertaken by the simulations in this paper. This requires the hydrodynamic model to be extended to allow roll and sway as well as surge, heave and pitch, with variable heading. This has been converted to state-space form and LNOC+AR control has been applied, for the first time for such a complex system with general heading. The objective is to demonstrate what capture widths may be achieved and to compare with idealised theoretical considerations.

Section 2 describes the linear diffraction-radiation model in state-space form. Section 3 describes how linear noncausal optimal control with auto-regression is applied. Section 4 presents numerical results. There is a discussion in Section 5 and conclusions are made in Section 6.

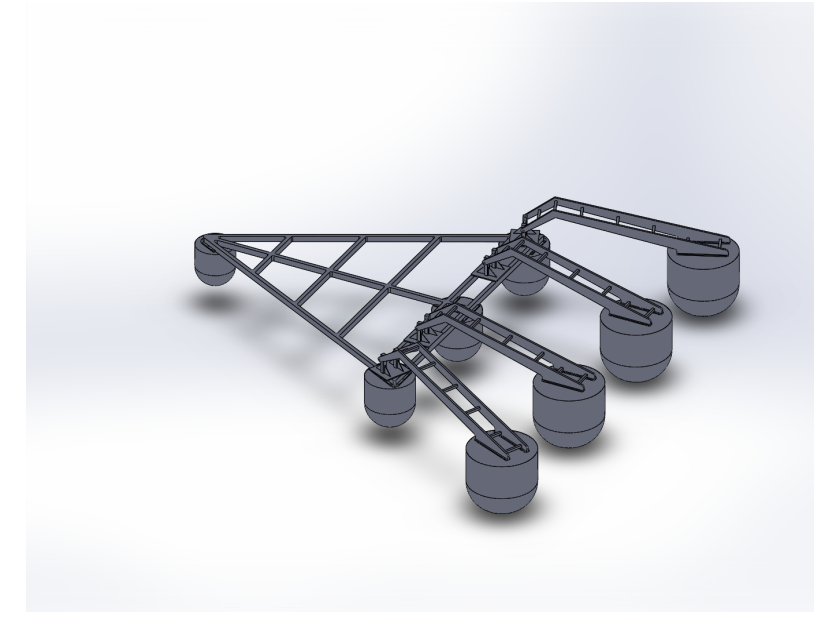

Fig. 1. 3-D view of the 8 floats 1-3-4 M4 configuration.

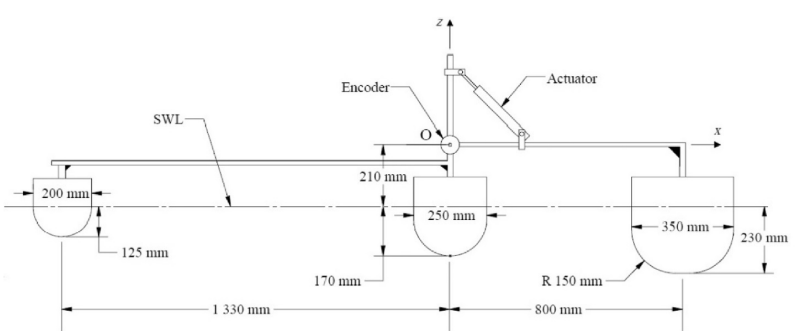

Fig. 2. Side elevation of laboratory scale M4 WEC. O is at the height of the hinge points of the PTOs.

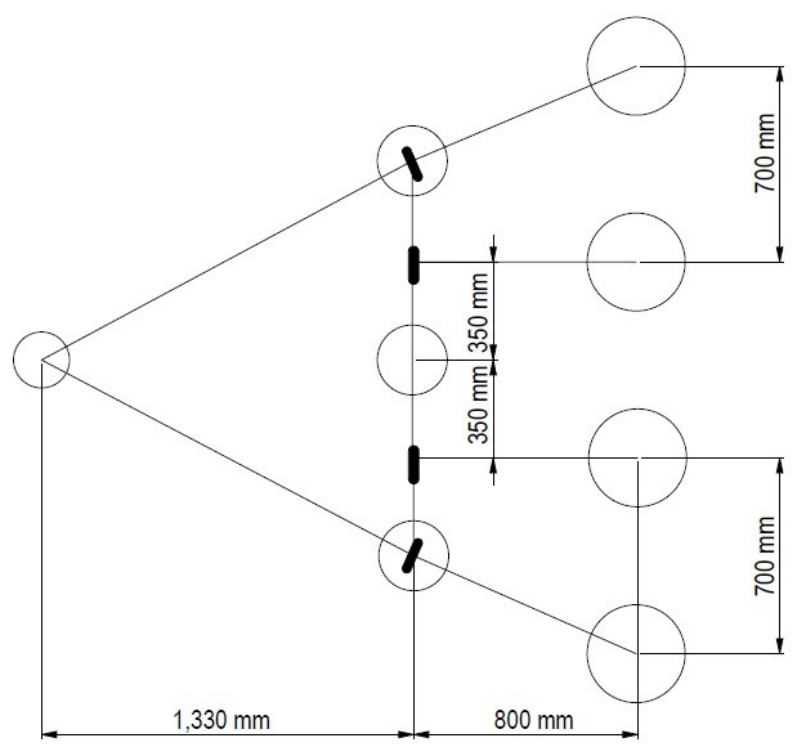

Fig. 3. 8-float 1-3-4 M4 WEC plan view at laboratory scale. The four thick lines denote the hinges for PTO.

\section{Linear Diffraction Model in State-Space Form}

The linear diffraction-radiation time-domain model formulated in [10] to include roll and sway, as well as surge, heave and pitch, for the 1-3-2 case has been extended to the 8-float 1-3-4 case. Different wave headings determine the excitation force. The hydrodynamic model of this 1-3-4 M4 is now 
converted to a state-space model for controller design based on the methodology of [6] for the 1-1-1 case.

A 3-D view of the configuration is shown in Fig.1 and the mathematical model is set up for laboratory scale, as the 1-1-1 and 1-3-2 cases, with dimensions as shown in Fig.2 for the side elevation and Fig.3 for the plan view. For such modelling Froude scaling may be applied to convert laboratory scale to full scale, demonstrated experimentally in [4], and here we are mainly concerned with non-dimensional capture width or capture width ratio.

In Fig. 3 , the eight floats are indexed by $i=1,2, \ldots, 8$ from left to right and from bottom to top, respectively. Coordinate origin $O$ is at the hinge height above the mid float $(i=3)$ with $x$ (surge) pointing towards right, $y$ (sway) upwards and $z$ (heave) outwards from the paper. Each float $(i)$ has five degrees of freedom (DOF): surge, sway, heave, roll, pitch. Incoming wave angle is fixed and thus yaw can be neglected. For a float $i, x_{i}, y_{i}, z_{i}$ are used to denote surge, sway and heave. Roll, pitch with respect to the WEC origin $O$ are denoted by $\psi_{i}$, $\theta_{i}$.

The generalized motion vector of the WEC can be chosen as $q=\left[x_{o}, y_{o}, z_{o}, \psi_{o}, \theta_{l}, \theta_{5}, \theta_{6}, \theta_{7}, \theta_{8}\right] . \theta_{l}$ is the pitch motion for the first four floats. $\theta_{l}$ equals $\theta_{i}$ for $i=1,2,3,4$ since they are one rigid body. $\theta_{5}, \theta_{6}, \theta_{7}, \theta_{8}$ are pitch motion of floats $5,6,7,8$. All floats have the same roll motion $\psi_{o}$.

Denote $h_{i}, t_{i}$ and $v_{i}$ the horizontal, transversal and vertical distance from the gravitational center of float $i$ to origin $O$. The translational motion of each float can be calculated by the following formulae, assuming small angle linearisation:

$$
\begin{aligned}
x_{i} & =x_{o}+h_{i}-\theta_{i} v_{i} \\
y_{i} & =y_{o}+t_{i}+\psi_{o} v_{i} \\
z_{i} & =z_{o}+v_{i}-\theta_{i} h_{i}+\psi_{o} t_{i}
\end{aligned}
$$

The motion equation for the 8-float M4 can be derived using Lagrangian equation as for the 3-float M4 [6] or by applying Newton's second law at each DOF and merge into matrix form. The motion equation is in the following form:

$$
M \ddot{q}(t)=f_{e, q}(t)+f_{r d, q}(t)+f_{r s, q}(t)+f_{p t o, q}(t)
$$

In this equation, $M$ is the mass and inertia matrix of size $9 \times 9$. $m_{i}$ applies to masses associated with each float 1-4, including float, ballast and beam (given in Table 1 below), and terms with $\sum m_{5-8}$ also include associated masses of float, ballast and beam for floats 5-8. $I_{\psi}$ denotes the inertia of roll of the WEC. $I_{1234}$ denotes the inertia of floats 1 to 4 in pitch and $I_{5}$ to $I_{8}$ denote those of the other floats. All inertias are relative to the origin $O . \ddot{q}$ is the second derivative of $q$. On the right hand side there are linear wave forces including excitation force, radiation damping force, hydrostatic restoring force and the controllable PTO torque, respectively. Mooring force in the experiment and model for 6-float M4 caused the horizontal motion to be less than $1 \mathrm{~cm}$ [5], which has negligible effect on phase in this model.

Hydrodynamic coefficients for calculating linear wave forces are obtained from WAMIT software [12]. These include all cross-coupled terms among floats.
Excitation force is independent of the WEC motions. In WAMIT 200 equally spaced frequencies from $0.02 \mathrm{~Hz}$ to $4 \mathrm{~Hz}$ are set as input, so that the excitation force coefficients including amplitude $F_{e x}$ and phase $\phi$ are of size 200 for each DOF of each float. For six DOFs and eight floats they are of size $200 \times 48$. Note we include 6 DOFs for generality although only 5 are applied. In simulations the JONSWAP wave spectrum is used with peakedness factor 1 and 3.3 to generate irregular wave profile covering the range of ocean conditions, giving amplitude in time domain $H$ and a random phase $\phi_{\text {ran }}$ of size 200 with the same frequency range as in WAMIT. Denote motion in each DOF as mode from $j=1, \ldots, 6$. Thus, excitation force for float $i$ in mode $j$ can be calculated as:

$$
\begin{array}{r}
f_{e, i, j}=\sum_{n=1}^{200} H(n) F_{e x}(n, 6(i-1)+j) \\
\cos \left(\phi(n, 6(i-1)+j)+\phi_{\text {ran }}(n)\right)
\end{array}
$$

Then these are rearranged to fit in the motion equation (4) as a $9 \times 1$ vector using equation (6).

$$
f_{e, q}(t)=\left[\begin{array}{c}
\sum_{i=1}^{8} f_{e, i, 1} \\
\sum_{i=1}^{8} f_{e, i, 2} \\
\sum_{i=1}^{8} f_{e, i, 3} \\
\sum_{i=1}^{8} f_{e, i, 4}-\sum_{i=1}^{8} f_{e, i, 2} v_{i}+\sum_{i=1}^{8} f_{e, i, 3} t_{i} \\
\sum_{i=1}^{4} f_{e, i, 5}+\sum_{i=1}^{4} f_{e, i, 1} v_{i}-\sum_{i=1}^{4} f_{e, i, 3} h_{i} \\
f_{e, 5,5}+f_{e, 5,1} v_{5}-f_{e, 5,3} h_{5} \\
f_{e, 6,5}+f_{e, 6,1} v_{6}-f_{e, 6,3} h_{6} \\
f_{e, 7,5}+f_{e, 7,1} v_{7}-f_{e, 7,3} h_{7} \\
f_{e, 8,5}+f_{e, 8,1} v_{8}-f_{e, 8,3} h_{8}
\end{array}\right]
$$

For surge, sway and heave DOFs the force at hinge $O$ is the sum of forces at each float. However, roll and pitch moments at $O$ are the moments at each float plus the moments induced by forces at each float. Note that in equation (6) all ' $f$ 's are functions of time.

Radiation damping force and hydrostatic restoring force are dependent on the WEC motion. Instead of calculating forces for each float and then rearranging them, coefficients are converted from $48 \times 48$ to $9 \times 9$ first, which makes the statespace form more straightforward. These equations for doing these are not shown here but readers can refer to [6] for more details.

Radiation damping force coefficients consist of two frequency dependent parts: the added mass matrix $A_{r d}$ and the radiation damping matrix $B_{r d}$. A standard way to deal with the added mass term is taking the value at infinite frequency as an approximation [13], which gives a constant matrix of size $48 \times 48$. Then it can be converted to size $9 \times 9$ and then added into $M$. The added mass matrix is denoted by $A_{\text {inf }}$.

The radiation damping matrix is of size $48 \times 48$ but in each element it is a frequency dependent sequence. This can be transferred into a impulse response function (IRF) as

$$
L_{m, n}(t)=\frac{2}{\pi} \int_{0}^{\infty} B_{r d, m, n}(\omega) \cos (\omega t) d \omega
$$

where $m, n=1, \ldots, 48$. There are $48 \times 48$ IRFs in total. The radiation damping force can be expressed using Cummins method [14] as a convolution of these IRFs and velocities. The 
time domain IRFs matrix $L$ can be converted to a $9 \times 9$ matrix $F_{r d}$. The equation for calculating radiation force is

$$
f_{r d, q}(t)=\int_{-\infty}^{t} F_{r d}(t-\tau) \dot{q}(\tau) d \tau
$$

The integral range can be set to several times the peak period with sufficient accuracy of the memory effect of radiation damping [5]. In this case the lower limit is $t-4 T_{p}$ where $T_{p}$ denotes the peak period of wave spectrum. The radiation damping forces in equation (8) can thus be expressed in a state-space form as:

$$
\begin{aligned}
\dot{z}_{s} & =A_{s} z_{s}+B_{s} \dot{q}(t) \\
f_{r d, q}(t) & =C_{s} z_{s}+D_{s} \dot{q}(t)
\end{aligned}
$$

where $\dot{z}_{s}$ is the state variable of the identified and assembled system and has no physical meaning. $A_{s}, B_{s}, C_{s}, D_{s}$ are the state-space matrices.

Hydrostatic restoring force is dependent on the heave displacement and roll, pitch rotation, but not on surge or sway. The heave stiffness of a float is $s_{z, i}=-\rho g \pi r_{i}^{2}$, and the pitch stiffness due to rotation is $10.97 \mathrm{Nm} / \mathrm{rad}$ for floats $1-4$ combined and $-7.22 \mathrm{Nm} / \mathrm{rad}$ for each float 5,6,7,8, accounting for weight and buoyancy forces and water plane restoring moment, where $\rho$ is the water density, $g$ is the gravitational constant and $r_{i}$ is the radius of float $i$. The roll stiffness for the combined floats is $-20.9 \mathrm{Nm} / \mathrm{rad}$. The heave stiffness is the dominant effect. The generalized hydrostatic restoring force can be written in a matrix form

$$
f_{r s, q}(t)=K q(t)
$$

where $K$ is a $9 \times 9$ hydrostatic restoring stiffness matrix. By combining the above equations, the motion equation for M4 can be rewritten as,

$$
\begin{aligned}
\left(M+A_{\infty}\right) \ddot{q}(t)+f_{r d, q}(t)+K q(t) & =f_{e, q}(t)+f_{p t o, q}(t) \\
\dot{z}_{s} & =A_{s} z_{s}+B_{s} \dot{q}(t) \\
f_{r d, q}(t) & =C_{s} z_{s}+D_{s} \dot{q}(t)
\end{aligned}
$$

To express the linear diffraction model in state-space form, we introduce a new state vector $x:=\left[q, \dot{q}, z_{s}\right]^{\top}$, so that the state-space representation of the 8-float M4 control-oriented model can be written as

$$
\begin{aligned}
& \dot{x}=A x+B f_{e, q}(t)+B f_{p t o, q}(t) \\
& y=C x+D u
\end{aligned}
$$

where the system matrices are

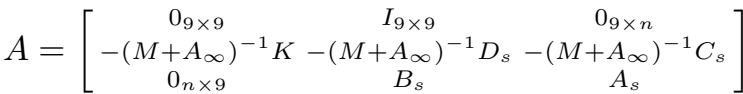

$$
\begin{aligned}
& B_{w}=\left[\begin{array}{c}
0_{9 \times 9} \\
\left(M+A_{\infty}\right)^{-1} \\
0_{n \times 9}
\end{array}\right] \\
& B_{u}=\left[\begin{array}{c}
0_{9 \times 1} \\
\left(M+A_{\infty}\right)^{-1}[0,0,0,0,1,-1,-1,-1,-1]^{\top} \\
0_{n \times 1}
\end{array}\right] \\
& C=\left[\begin{array}{ccccccc}
0_{1 \times 13} & 1 & -1 & 0 & 0 & 0 & 0_{1 \times n} \\
0_{1 \times 13} & 1 & 0 & -1 & 0 & 0 & 0_{1 \times n} \\
0_{1 \times 13} & 0 & 0 & -1 & 0 & 0_{1 \times n} \\
0_{1 \times 13} & 1 & 0 & 0 & 0 & -1 & 0_{1 \times n}
\end{array}\right]
\end{aligned}
$$

Without control, the PTO torques are modelled as four passive dampers with the same damping constant $d_{k}$. Note that the value of $d_{k}$ is selected by trail and error for best power capture performance.

\section{Noncausal Optimal Controller Design}

The linear noncausal optimal controller (LNOC) was developed to tackle the WEC energy maximizing problem [15], and its efficacy was demonstrated by a simple heaving point absorber showing remarkable power capture improvements. In [6], the LNOC was applied to the 3-float M4. The power capture was shown to be increased by $40 \%$ to $100 \%$ depending on peak periods.

In this section, the LNOC controller in mathematical form is briefly introduced. For more details on the theories behind the controller readers can refer to [15].

With the control-oriented model presented in the last section, we can formulate the optimal control problem as follows:

$$
\min _{u_{0}, \ldots, u_{N}} \Sigma_{k=0}^{N}\left\{z_{k} u_{k}+\frac{1}{2} x_{k}^{\top} Q x_{k}+\frac{1}{2} R u_{k}^{2}\right\}
$$

subject to the discrete-time state-space model

$$
\begin{aligned}
x_{k+1} & =A x_{k}+B_{w} w_{k}+B_{u} u_{k} \\
z_{k} & =C x_{k}
\end{aligned}
$$

which is discretized from the continuous time model (12). Here $w_{k}$ is the wave excitation force at time $k$ and $u_{k}$ is the control input at time $k$.

The control law derived from resolving this optimal noncausal control problem has the form of

$$
u_{k}=K_{x} x_{k}+K_{d} w_{k, n p}
$$

which consists of a feedback term for the system states $x_{k}$ and a feed-forward term to incorporate the prediction of the incoming wave excitation force $w_{k, n p}:=\left[w_{k}, w_{k+1}, \ldots, w_{k+n_{p}-1}\right]$ where $n_{p}$ is the length of wave prediction horizon. The controller (19) is noncausal because its current control input depends not only on the current system states information $x_{k}$ but also on the future incoming wave information $w_{k, n p}$. The importance of incorporating this noncausal information into control has been demonstrated in [15].

$K_{x}$ and $K_{d}$ are constant coefficient matrices that can be pre-calculated off-line. The formulae for calculating them are

$$
\begin{aligned}
K_{x} & =-\left(R+B_{u}^{\top} V B_{u}\right)^{-1}\left(C+B_{u}^{\top} V A\right) \\
K_{d} & =-\left(R+B_{u}^{\top} V B_{u}\right)^{-1} B_{u}^{\top} \Psi
\end{aligned}
$$

and

$$
\begin{gathered}
V=Q+A^{\top} V A-\left(C+B_{u}^{\top} V A\right)^{\top} \\
\left(R+B_{u}^{\top} V B_{u}\right)^{-1}\left(C+B_{u}^{\top} V A\right)
\end{gathered}
$$

where $V$ is the solution of the algebraic Ricatti equation (22). Let $\Phi:=\left(A+B_{u} K_{x}\right)^{\top}$, then $\Psi:=$ $\left[V B_{w}, \Phi V B_{w}, \ldots, \Phi^{n_{p}-1} V B_{w}\right]$.

The control law (19) implies that for optimal power capture, knowledge of the incoming wave force amplitudes are required. This can be realized by external wave force prediction 
techniques, e.g. the deterministic sea wave prediction (DSWP) technology [16]. It has also been found that incoming wave force predictions can be realized by combining a simple auto-regressive (AR) prediction model and a Kalman filter to observe the excitation forces based on measurements of WEC motions [7]. This realistic solution makes incoming wave force prediction viable. Numerical results show that the performance degradation is relatively small compared to the ideal wave predictions case. In this paper, control performances are demonstrated for both ideal predictions and predictions realized by the AR model.

\section{Numerical Simulations}

Numerical simulations are carried out to demonstrate modelling fidelity and control performances. These are presented in two parts: zero degrees heading angle and non-zero degrees heading angles. For both cases, simulations are run by Matlab on a PC with Inter Core i5-4670K CPU $(3.4 \mathrm{GHz})$. Sampling time is set dependent on peak period $T_{s}=T_{p} / 200$ to maintain 200 time steps run in each peak period. For one simulation 100 peak periods are run. For each specific sea state one simulation is completed within ten minutes.

JONSWAP wave spectra with peakedness factor of 1 and 3.3 are both used to generate wave profiles. Significant wave height is fixed at $H_{s}=0.04 \mathrm{~m}$ although this is normalized by the performance index capture width ratio (CWR) The capture width ratio is normalized by the wavelength corresponding to the frequency at the centroid of the wave spectrum. Previous study shows that this definition collapses CWR for JONSWAP waves with different $\gamma$ [11]. Peak periods range from $T_{p}=0.7 s$ to $1.8 s$, each with $0.1 s$ interval. For Froude scaling these are 4.95 to 12.7 seconds in full scale which is typically 50 times the model scale. These wave profiles are fed as disturbances to the WEC model and WEC responses are recorded to calculate absorbed energy. For fair comparison to a passive damper only one set of control parameters $Q$ and $R$ are used for all wave conditions. The passive damping ratio $d_{k}=3 N M s / r a d$, identical for 4 PTOs.

Mass and position of centre of mass relative to the hinge point origin $O$ for each mechanical part of the 1-3-4 M4 are shown in Table I. Inertia in pitch of the first 4 floats is $I_{1234}=5.58 \mathrm{kgm}^{2}$. Inertia in pitch of each stern float is $I_{5}=I_{6}=I_{7}=I_{8}=13.46 \mathrm{kgm}^{2}$. Inertia in roll of the whole WEC is $I_{\psi}=56.51 \mathrm{kgm}^{2}$. These are used in the state-space model for numerical simulations. The natural period for heave motion of each float is: $0.75 \mathrm{~s}$ for the bow float, $0.76 \mathrm{~s}$ for the mid floats and $1.03 \mathrm{~s}$ for the stern floats, taking account of the added mass. For pitch about $O$ the natural period of bow-mid floats is close to $0.75 \mathrm{~s}$, of stern floats are close to $1.03 \mathrm{~s}$. Note that the natural periods are thus within the range of selected JONSWAP peak periods. In addition the bow and mid, and mid and stern, floats should be predominantly in antiphase to generate relative pitch about $\mathrm{O}$. The mid-stern spacing is about half a wavelength for a wave period of $1 \mathrm{~s}$ and it was found advantageous for the bow-mid spacing to be rather larger, increasing the moment arm about $\mathrm{O}$, corresponding to a wave period of about $1.3 \mathrm{~s}$.
TABLE I

MASS AND CENTRE OF MASS

\begin{tabular}{|c|c|c|c|c|c|}
\hline Part & mass $(\mathrm{kg})$ & $\mathrm{h}(\mathrm{m})$ & $\mathrm{t}(\mathrm{m})$ & $\mathrm{v}(\mathrm{m})$ & radius $(\mathrm{m})$ \\
\hline float 1 & 1.2 & -1.33 & 0 & -0.222 & 0.1 \\
\hline ballast 1 & 1.0 & -1.33 & 0 & -0.335 & \\
\hline beam 1234 & 0.289 & -0.7 & 0 & 0.094 & \\
\hline float 2 & 1.887 & 0 & -0.7 & -0.214 & 0.125 \\
\hline ballast 2 & 3.0 & 0 & -0.7 & -0.327 & \\
\hline float 3 & 1.887 & 0 & 0 & -0.214 & 0.125 \\
\hline ballast 3 & 3.0 & 0 & 0 & -0.327 & \\
\hline float 4 & 1.887 & 0 & 0.7 & -0.214 & 0.125 \\
\hline ballast 4 & 3.0 & 0 & 0.7 & -0.327 & \\
\hline float 5 & 3.74 & 0.8 & -1.05 & -0.256 & 0.175 \\
\hline ballast 5 & 13.5 & 0.8 & -1.05 & -0.354 & \\
\hline beam 5 & 0.56 & 0.5 & -1.05 & 0 & \\
\hline PTO 1 & 0.19 & 0.16 & -1.05 & -0.16 & \\
\hline float 6 & 3.74 & 0.8 & -0.35 & -0.256 & 0.175 \\
\hline ballast 6 & 13.5 & 0.8 & -0.35 & -0.354 & \\
\hline beam 6 & 0.56 & 0.5 & -0.35 & 0 & \\
\hline PTO 2 & 0.19 & 0.16 & -0.35 & -0.16 & \\
\hline float 7 & 3.74 & 0.8 & 0.35 & -0.256 & 0.175 \\
\hline ballast 7 & 13.5 & 0.8 & 0.35 & -0.354 & \\
\hline beam 7 & 0.56 & 0.5 & 0.35 & 0 & \\
\hline PTO 3 & 0.19 & 0.16 & 0.35 & -0.16 & \\
\hline float 8 & 3.74 & 0.8 & 1.05 & -0.256 & 0.175 \\
\hline ballast 8 & 13.5 & 0.8 & 1.05 & -0.354 & \\
\hline beam 8 & 0.56 & 0.5 & 1.05 & 0 & \\
\hline PTO 4 & 0.19 & 0.16 & 1.05 & -0.16 & \\
\hline
\end{tabular}

Centre of mass is relative to hinge point origin $O . \mathrm{h}$ (horizontal) is for $\mathrm{x}$ axis; $\mathrm{t}$ (transversal) is for $\mathrm{y}$ axis; $\mathrm{v}$ (vertical) is for $\mathrm{z}$ axis. Beam 1234 connect bow and mid floats. Radius is of circular cross section.

\section{A. Zero degrees heading}

Fig.4 shows the capture width ratio (CWR) plot for different peak periods. This can be viewed equivalently as normalised power capture and enables comparison to some idealised optimum value for point absorbers and hinged rafts in regular waves [17], [18]. Note that the CWR is independent of significant wave height.

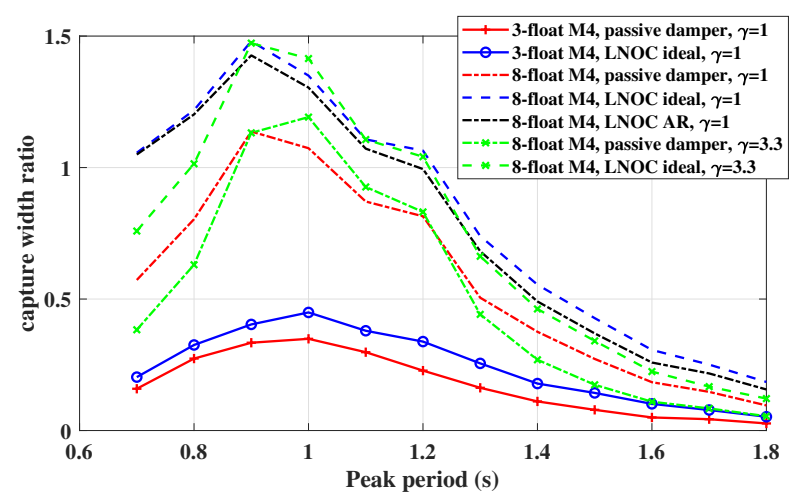

Fig. 4. Capture width ratio, significant wave height $H_{s}=0.04 m$, zero degrees heading. Comparing the 3-float M4 CWR which was validated by experimental data with the 8 -float M4 CWR derived by simulations.

In Fig.4 the solid line with ' + ' sign shows the CWR of 3float M4 with a passive damper which has been validated by experiment data. This provides justification for the fidelity of the 8-float M4 model as no experiment has been carried out yet. It can be seen that without control, the maximum CWR is 
0.35 for the 3-float M4 with 1 PTO. For the 8-float M4 with 4 PTOs this is 1.13 , slightly less than 4 times of the 3 -float case.

The LNOC is applied to the 8-float M4 with incoming wave predictions. It can be seen that with LNOC and ideal prediction, the CWR at $T p=0.9 \mathrm{~s}$ increases from about 1.13 to about 1.47 which is $30 \%$ improvement. At $T p=1.8 s$ this improvement goes up to $93 \%$. The average improvement for all peak periods is around $53 \%$. The CWRs of 3-float M4 with passive damper and LNOC are also presented at the bottom. Control performances are similar for both WECs.

The AR model with non-perfect wave force predictions (black dash line) brings performance degradation but improvements of capture width are still significant compared to the passive damper case, in this case from $21 \%$ to $83 \%$. These are run with a JONSWAP spectra with $\gamma=1$.

To demonstrate that the LNOC also works in a narrow band spectrum, the JONSWAP spectra with $\gamma=3.3$ is also shown in Fig.4 as the dash line with " $x$ " symbol. The control performance is similar to the $\gamma=1$ case.

Fig.5 to Fig.6 show the time responses at $T_{p}=1.0 \mathrm{~s}$, comparing the passive damper and LNOC with ideal wave force predictions and AR predictions. These include the PTO torque (control input) and the power capture for all the PTOs. It is clear that at zero degrees heading all PTOs are operating in parallel and their responses are almost identical. This shows the LNOC is able to control 4 PTOs simultaneously and enables the same power capture improvement for all of them.
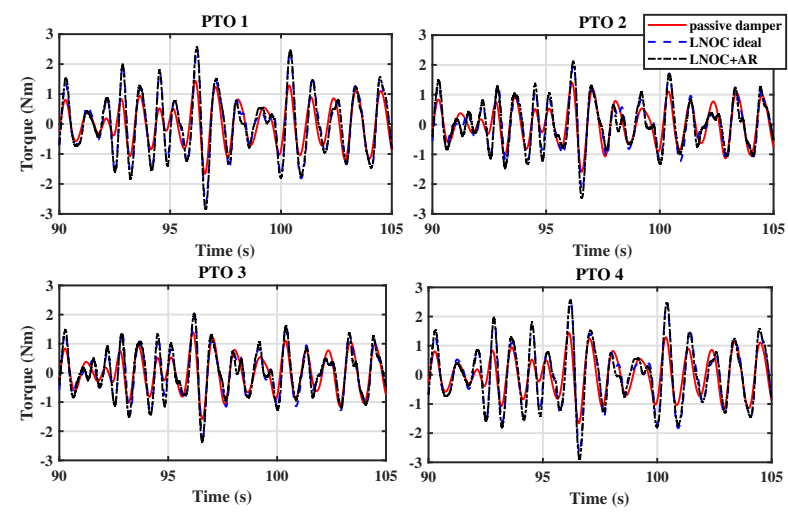

Fig. 5. PTO control torque, significant wave height $H_{s}=0.04 m$, peak period $T_{p}=1.0 s, \gamma=1$, zero degrees heading.

\section{B. Non-zero headings}

Non-zero headings generally decrease the CWR of M4 WECs. In this part simulation results of passive damper and LNOC under different WEC headings are compared. For different heading angles ranging from 0 degrees to 90 degrees the excitation force coefficients $F_{e x}$ and $\phi$ are changed. These coefficients are pre-calculated by WAMIT. Other hydrodynamic coefficients were not changed.

Fig.7 and Fig.8 shows the CWR trends of passive damper and LNOC for $T_{p}=1.0 \mathrm{~s}$ when WEC headings change from 0 degrees to 90 degrees, respectively for $\gamma=1$ and $\gamma=3.3$.
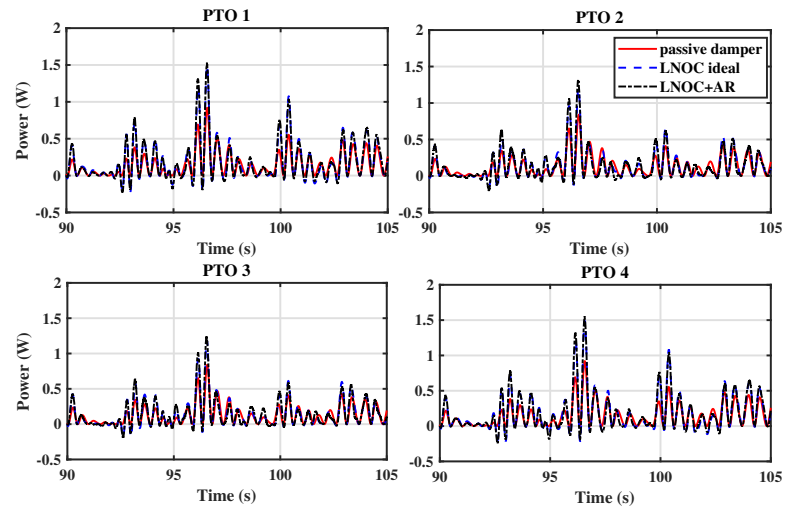

Fig. 6. Instant power, significant wave height $H_{s}=0.04 m$, peak period $T_{p}=1.0 s, \gamma=1$, zero degrees heading.

Orange lines show rotation angles of roll motion and the RMS pitch angles for 4 PTOs in average.

It can be observed that as incoming wave angle increases from zero, the relative pitch angle decreases, so do the CWRs. The roll motion of the whole WEC increases from almost zero at zero heading to a significant value compared with pitch at 20 degrees heading and then stays relatively stable.

The LNOC controller, for both ideal wave force prediction and AR cases, can improve CWR for all WEC heading angles. Surprisingly, the improvements for non-zero headings are more significant than for zero heading.

In a more realistic scenario, the WEC heading is likely to fluctuate around zero as wind and current exist. This result with unchanged control parameters ensures the LNOC can be applied and improve power capture of the WEC regardless of change of the WEC headings.

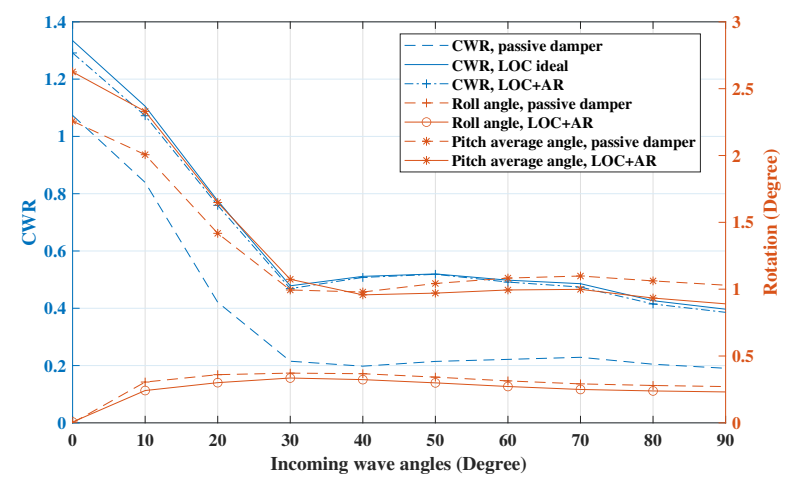

Fig. 7. Left $Y$ axis: CWR of passive damper and LNOC. Right $Y$ axis, rotation motion angle (RMS value) of pitch and roll. $X$ axis wave heading angle from 0 degrees to 90 degrees. Significant wave height $H_{s}=0.04 m$, peak period $T_{p}=1.0 \mathrm{~s}, \gamma=1$.

\section{Tuning of control parameters}

Note that in the previous sections control parameters Q and $\mathrm{R}$ are particularly tuned for zero-headings. For fair comparison against a passive damper these parameters are not changed for non-zero headings.

However, using distinct parameters for various sea states (peak periods, headings) enables even higher improvement of 


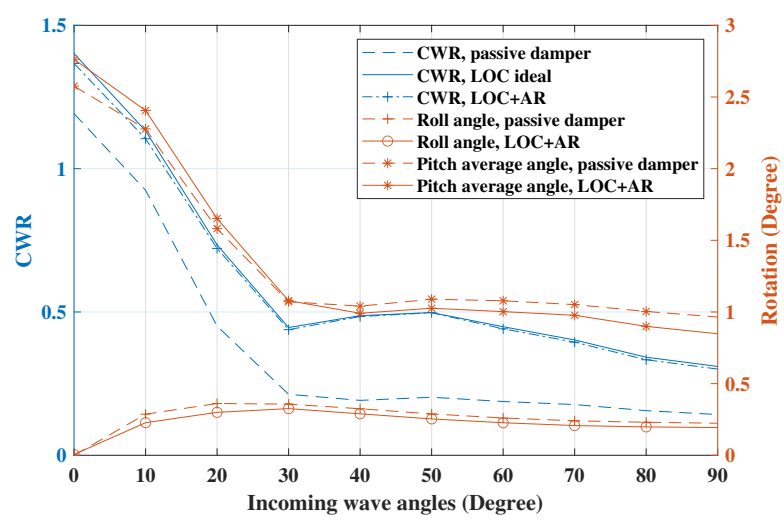

Fig. 8. Left $Y$ axis: CWR of passive damper and LNOC. Right $Y$ axis, rotation motion angle (RMS value) of pitch and roll. $X$ axis wave heading angle from 0 degrees to 90 degrees. Significant wave height $H_{s}=0.04 m$, peak period $T_{p}=1.0 s, \gamma=3.3$.

the CWR. Fig.9 shows the benefit of adopting distinct headingdependent control parameters instead of using uniform ones. Control parameters are specifically tuned for peak periods $1.0 s, 1.2 s, 1.4 s, 1.8 s$ and WEC headings of $0,10,20,40$, 60,90 degrees to cover the physical parameter range. At 0 degrees heading the control parameters were the same as in the previous sections. From 20 degrees, higher CWR can be observed by using distinct parameters (purple line) compared to the uniform tuning one (orange line). Interestingly, at peak period $1.4 \mathrm{~s}$ and $1.8 \mathrm{~s}$, for non-zero degrees heading, with specifically tuned LNOC, the CWR is higher than that at zerodegree heading. Note that at 90 degrees the four PTOs are working predominantly in heave so similar to point absorbers.

Fig.10 shows average power for same conditions as Fig.9. The relative effects for each period and heading are the same but it can be seen that power is more uniform than CWR varying between 0.5 and $1.6 \mathrm{~W}$ across all conditions with distinct control parameters. These results further reveal the potential of the LNOC.

Implementing the LNOC with various parameters depending on the operating sea conditions instead of using uniform ones requires an adaptive mechanism. In practice, this can be realized by pre-tuning the corresponding control parameters for various headings/peak periods and storing them in an onboard lookup table. As the sea condition changes the control would recognize and switch parameters accordingly.

\section{DISCUSSION}

The analysis has been based on linear diffraction-radiation modelling. This has previously been compared with experiments with linear damper PTO for the 1-1-1 and 1-3-2 cases in relatively large, though different, wave basins. The power capture in operational conditions was slightly under-predicted in the 1-3-2 case. The reason is not yet understood and nonlinear effects, such as drag, are generally thought to reduce, not increase, power capture, e.g. [19]. In a wave basin the wavemakers generally absorb reflected waves but there are inevitable reflections from the beaches which result directly from the generated waves and also from waves radiated from
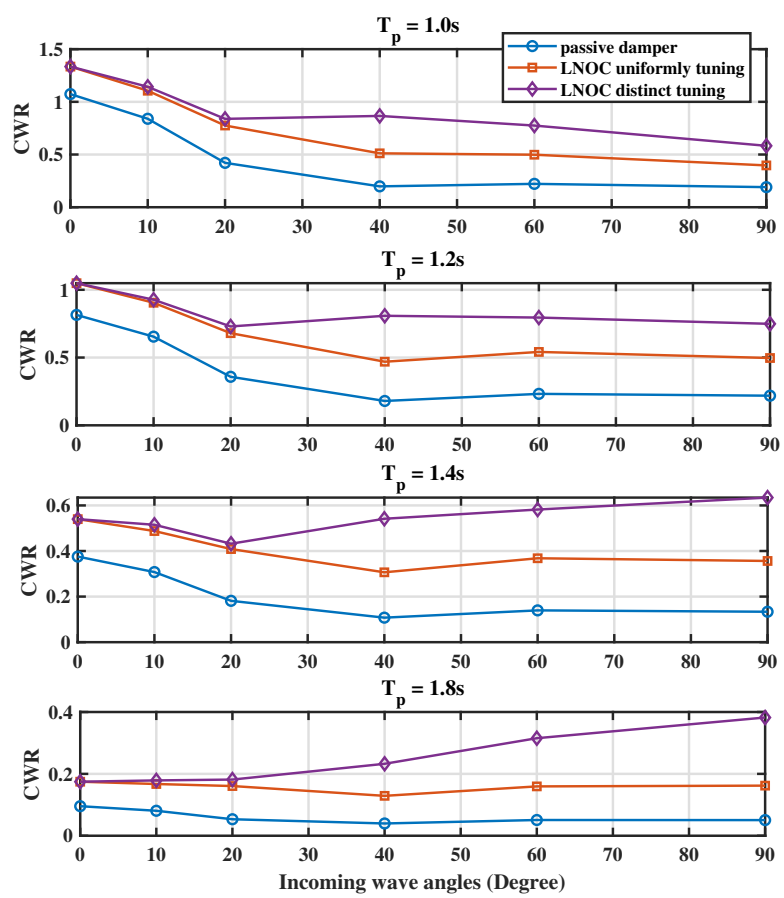

Fig. 9. Capture width ratio comparing LNOC with uniformly tuning and distinct tuning for different sea conditions. From top to bottom plot peak periods are $1.0 s, 1.2 s, 1.4 s$ and $1.8 s$. Data are obtained for $0,10,20,40$, 60 and 90 degrees heading. Significant wave height $H_{s}=0.04 m, \gamma=1$.
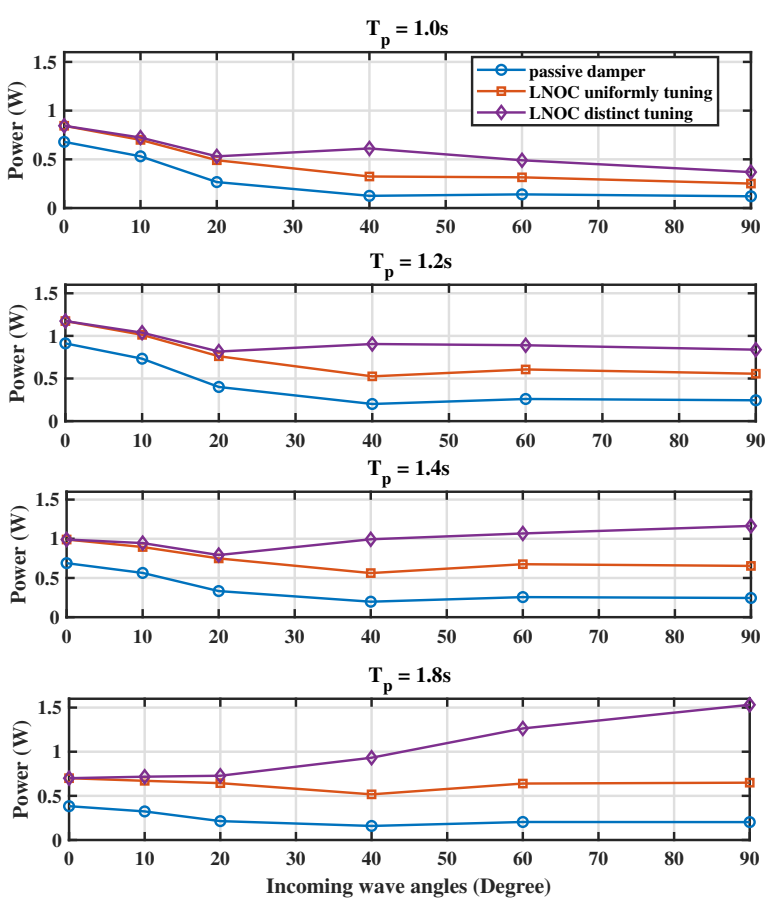

Fig. 10. Average captured power (sum of all PTOs), comparing LNOC with uniformly tuning and distinct tuning for different sea conditions. From top to bottom plot peak periods are $1.0 \mathrm{~s}, 1.2 \mathrm{~s}, 1.4 \mathrm{~s}$ and $1.8 \mathrm{~s}$. Data are obtained for $0,10,20,40,60$ and 90 degrees heading. Significant wave height $H_{s}=$ $0.04 m, \gamma=1$.

the body motions and radiated wave power is of similar magnitude to that absorbed [10]. The analysis of power capture is conservative based on empirical evidence. In extreme waves 
without mechanical damping the response was found to be remarkably linear based on experiments in focussed waves for a different 1-1-1 case [9] and time domain linear modelling in extreme irregular waves gave good predictions for the 1-32 case, improved by the addition of a small drag coefficient in relatively large motion [10]. A small drag coefficient has also been predicted for these streamlined shapes through computational fluid dynamics (CFD) in [20]. Since control generally improves power capture by causing greater motion it is important to know that linear hydrodynamics still apply. It should be noted that, while angular response was effectively linear, the mooring loads are highly nonlinear in extreme waves. Optimum design for moorings is in progress [21].

The aim has been to show that high capacity wave energy systems are possible by capturing energy from multiple floats with multi-mode forcing, as incorporated in the M4 configuration. It is useful to compare capture widths with the maximum possible for point absorbers and hinged beam rafttype absorbers, available for regular waves at resonance. For point absorbers the maximum capture width ratio, normalised by wave length, is $1 / 2 \pi$ for heave, $1 / \pi$ for surge and pitch and $3 / 2 \pi$ in combination [17]. For the hinged beam it is $4 / 3 \pi$ [18]. In the 1-3-4 case here we have an attenuator or multiple line absorbers with 4 PTOs. For zero heading, as intended, if we considered this as 4 hinged beams the maximum CWR would be 1.7 while a maximum value of 1.2 is achieved in irregular waves, 1.5 with control. If we considered 4 point absorbers operating in heave, surge and pitch the maximum value would be 1.9. If we considered this as 4 point absorbers and 4 hinged beams in combination then the maximum would be 3.6 . But this would require $3 \times 4=12$ floats and here we only use 8 . A crude estimate of theoretical maximum might thus be 2.4 . The intention is to compare with what is theoretically possible for power absorption. If this were to be called efficiency it would be around $62.5 \%$.

With different headings the contributions from each mode will be different. However for a heading of 90 degrees the 4 stern floats are responding mainly in heave and the maximum CWR would be 0.63 . With LNOC and distinct parameters this is achieved with $T_{p}=1.2 \mathrm{~s}$ and $1.4 \mathrm{~s}$ giving $100 \%$ efficiency while for $T_{p}=1 s$ and $1.8 s$ it is 0.55 and 0.4 giving efficiencies of $87 \%$ and $63 \%$. When converted into power, this is now greater for $T_{p}=1.8 \mathrm{~s}$ and $1.4 \mathrm{~s}$ than for $T_{p}=1.2 \mathrm{~s}$ and $1 \mathrm{~s}$. The system was designed for zero heading which will be the case for ocean conditions with small currents. With currents, e.g. on continental shelf regions, the situation is complex with variable heading and the optimum float configuration for maximum energy capture needs to be determined. For this configuration the average power is between 0.4 and $1.2 \mathrm{~W}$ corresponding to 0.35 and $1.1 \mathrm{MW}$ at 1:50 scale with $H_{s}=2 \mathrm{~m}$. This would relate to the average wave conditions at Wavehub mentioned in the introduction section.

The worldwide average wave power has been estimated to be about 2 TW [1] but this is not all available for conversion to electricity. For the theoretical idealised cases mentioned the absorbed power is equal to the power in radiated waves (good absorbers are good radiators). Thus only half the available wave power is converted. This was also estimated for the 13-2 M4 in [10] and found to be similar. The available average wave power for conversion is thus about $1 \mathrm{TW}$. Of course there are also many practical constraints, e.g. accessibility of sites, depth of water etc., but much infrastructure is similar to and could be shared with offshore wind farms.

\section{CONCLUSiON}

Wave energy converters may have capacity similar to wind turbines when based on multi-float configurations with multimode forcing. A particular hinged raft-type system, known as M4, with 8 floats and 4 PTOs is considered here. Control improves energy capture and this has not previously been applied to such complex systems with many freedoms. This has been possible in real time due to linear hydrodynamic modelling in a state-space form, previously validated experimentally. Linear non-causal optimal control with autoregressive wave force prediction is applied. For the design case with zero heading power capture is increased by $21-83 \%$. The maximum power capture in irregular waves is about $60 \%$ that for superimposed idealised components, in regular waves at resonance. Off design, as the heading angle increases from zero, power capture, though reduced as contributions from different modes of forcing vary, can be close to ideal for some peak periods with control parameters distinct for each heading. In areas with significant currents causing non-zero headings the float configuration for optimum energy capture would need to be reconsidered. The present design is intended for ocean conditions with insignificant currents.

\section{ACKNOWLEDGEMENT}

Zhijing Liao is grateful to the financial support from the joint "Queen Mary University of London - China Scholarship Council" scholarship.

Guang Li thanks the support of Wave Energy Control Systems Programme by Wave Energy Scotland.

\section{REFERENCES}

[1] K. Gunn and C. Stock-Williams. Quantifying the global wave power resource. Renewable Energy, 44:296 - 304, 2012.

[2] A. F. de O. Falcão. Wave energy utilization: A review of the technologies. Renewable and Sustainable Energy Reviews, 14(3):899 - 918, 2010.

[3] P. Stansby, E. Carpintero Moreno, T. Stallard, and A. Maggi. Threefloat broad-band resonant line absorber with surge for wave energy conversion. Renewable Energy, 78:132 - 140, 2015.

[4] P. Stansby, E. Carpintero Moreno, and T. Stallard. Capture width of the three-float multi-mode multi-resonance broadband wave energy line absorber $\mathrm{m} 4$ from laboratory studies with irregular waves of different spectral shape and directional spread. Journal of Ocean Engineering and Marine Energy, 1(3):287-298, Aug 2015.

[5] P. Stansby, E. Carpintero Moreno, and T. Stallard. Large capacity multifloat configurations for the wave energy converter $\mathrm{m} 4$ using a timedomain linear diffraction model. Applied Ocean Research, 68:53 - 64, 2017.

[6] Z. Liao, N. Gai, P. Stansby, and G. Li. Linear non-causal optimal control of an attenuator type wave energy converter m4. IEEE Transactions on Sustainable Energy, pages 1-1, 2019.

[7] Z. Liao, P. Stansby, and G. Li. A generic linear non-causal optimal control framework integrated with wave excitation force prediction for multi-mode wave energy converters with application to m4. Applied Ocean Research, 97:102056, 2020. 
[8] U. A. Korde. Near-optimal control of a wave energy device in irregular waves with deterministic-model driven incident wave prediction. Applied Ocean Research, 53:31 - 45, 2015.

[9] H. Santo, P. H. Taylor, E. Carpintero Moreno, P. Stansby, R. Eatock Taylor, L. Sun, and J. Zang. Extreme motion and response statistics for survival of the three-float wave energy converter $\mathrm{m} 4$ in intermediate water depth. Journal of Fluid Mechanics, 813:175-204, 2017.

[10] P. Stansby and E.Carpintero. Moreno. Hydrodynamics of the multifloat wave energy converter $\mathrm{m} 4$ with slack moorings: Time domain linear diffraction-radiation modelling with mean force and experimental comparison. Applied Ocean Research, 97:102070, 2020.

[11] E. Carpintero Moreno and P. Stansby. The 6-float wave energy converter $\mathrm{m} 4$ : ocean basin tests giving capture width, response and energy yield for several sites. Renewable and Sustainable Energy Reviews, 104:307 $-318,2019$.

[12] C.H. Lee and J. N. Newman. Wamit - user manual version 7.0. In WAMIT Inc, Chestnut Hill, Massachusetts, 2013.

[13] Z. Yu and J. Falnes. State-space modelling of a vertical cylinder in heave. Applied Ocean Research, 17(5):265 - 275, 1995.

[14] W.E.Cummins. The impulse response function and ship motions. 1962.

[15] S. Zhan and G. Li. Linear optimal noncausal control of wave energy converters. IEEE Transactions on Control Systems Technology, 27(4):15261536, July 2019.

[16] M. Belmont, J. Christmas, J. Dannenberg, Tyson Hilmer, J. Duncan, J.M. Duncan, and B. Ferrier. An examination of the feasibility of linear deterministic sea wave prediction in multidirectional seas using wave profiling radar: Theory, simulation, and sea trials. Journal of Atmospheric and Oceanic Technology, 31:1601-1614, 072014.

[17] J. Falnes. Ocean Waves and Oscillating Systems: Linear Interactions Including Wave-Energy Extraction. Cambridge University Press, 2002.

[18] J.N. Newman. Absorption of wave energy by elongated bodies. Applied Ocean Research, 1(4):189 - 196, 1979.

[19] G. Giorgi and J. V. Ringwood. Comparing nonlinear hydrodynamic forces in heaving point absorbers and oscillating wave surge converters. Journal of Ocean Engineering and Marine Energy, 4(1):25-35, Feb 2018.

[20] H. Gu, P. Stansby, T. Stallard, and E. Carpintero Moreno. Drag, added mass and radiation damping of oscillating vertical cylindrical bodies in heave and surge in still water. Journal of Fluids and Structures, 82:343-356, 102018

[21] P. Stansby and E. Carpintero Moreno. Study of snap loads for idealized mooring configurations with a buoy, inextensible and elastic cable combinations for the multi-float $\mathrm{m} 4$ wave energy converter. Water 12(10), 2020.

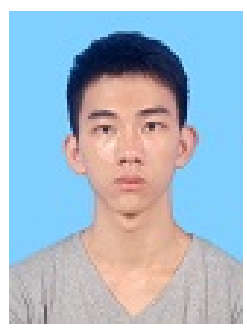

Zhijing Liao was born in Guangdong China in 1994 $\mathrm{He}$ received his double bachelor degree from Beijing Institute of Technology, Beijing, China, and Polytechnic University of Turin, Turin, Italy, in 2017. $\mathrm{He}$ is currently working toward the Ph.D. degree at Queen Mary University of London, London, U.K. His research interests include modelling and control of wave energy converters.

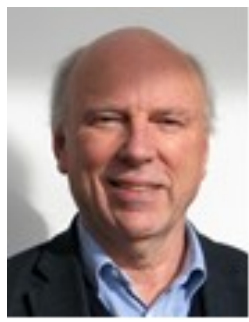

Peter Stansby Peter Stansby was awarded his Batchelor and $\mathrm{PhD}$ degrees by the University of Cambridge and a DSc by the University of Manchester. He is a Fellow of the Royal Academy of Engineering and currently the inaugural Osborne Reynolds Professor of Fluid Mechanics in the School of Engineering in the University of Manchester. His research interests have been in offshore and coastal hydrodynamics including computational and physical modelling. He now concentrates on offshore renewable energy and has been developing the multi-float wave energy converter M4 for a decade.

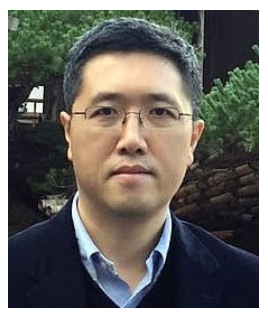

Guang Li (M'09) received his Ph.D. degree in Electrical and Electronics Engineering, specialized in control systems, from the University of Manchester, in 2007. He is currently a Senior Reader in dynamics modelling and control in Queen Mary University of London, UK. His current research interests include constrained optimal control, model predictive control, adaptive robust control and control applications including renewable energies and energy storage, etc.

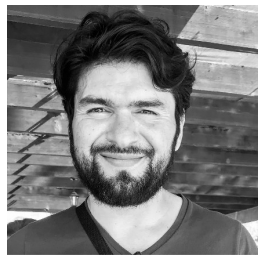

Efrain Carpintero Moreno completed his Ph.D. at the University of Manchester in 2015, focused on the development of WECs and Wind-Wave hybrid converter concepts. He became a research associate at the University of Manchester and in 2017 he was awarded a research grant from the national research council in Mexico (Conacyt) for energy capture at Mexican waters. In July 2020 he became a researcher and scientific consultant for the Blue Accelerator project based in Ghent University. He currently holds the chair for the Belgium committee preparing international standards for marine conversion system "IEC - TC114 Marine energy - Wave, tidal and other water current converters". 